\title{
Squeezing the Turnip with Artificial Neural Nets
}

\author{
Leonard J. Francl
}

Department of Plant Pathology, The Pennsylvania State University, University Park 16802.

Accepted for publication 23 June 2004.

\begin{abstract}
Francl, L. J. 2004. Squeezing the turnip with artificial neural nets. Phytopathology 94:1007-1012.

Modeling in epidemiology has followed many different strategies and philosophies. Artificial neural networks (ANNs) comprise a family of highly flexible and adaptive models that have shown promise for application to modeling disease phenomena in general and plant disease fore-

casting in particular. ANN modeling requires the availability of representative, robust input data and exhaustive testing of model aptness and optimization; meanwhile, ANNs sacrifice much of the biological insight often derived through other model forms. On the other hand, ANNs may extract previously undetected and possibly complex relationships, which can increase prediction accuracy over mainstream statistical methods, usually in an incremental manner.
\end{abstract}

Rationale for the application of artificial neural networks. Diverse modeling approaches have advanced understanding (i.e., predictability) of phenomena associated with disease in plant populations; moreover, empirical models have particular utility should one want to develop a disease forecasting system because field data are related patently to the forecasting application and one readily can validate model aptness. Also, physical limits and exceptions to model applicability can be revealed through careful analysis, whereas like conditions may never be encountered in a controlled environment and often may remain unanticipated. On the other hand, uncontrolled weather, which may or may not be suitable for disease development and in any event limits research to the cropping season, effectively wastes or at least prolongs resource utilization. Lastly, the physical world is inherently complex, so duplicated trials, potential range of variables, and certain combinations of factors are impossible to attain. In sum, field experimentation may prolong data collection but assessment of empirical model performance in the real world is an inclusive process.

The challenge of modeling disease severity under field conditions sparked my interest in artificial neural networks (ANNs), which are reputed to excel at extracting sometimes subtle patterns from large multivariate data sets without preconceived assumptions about model form due to incompletely understood, possibly complex, relationships. A major advantage of neural networks is that one usually can construct a multidimensional, nonlinear response surface without pre-specified derivatives, intercepts, or bounds. Further, a solution is possible for classification of binary events, such as an epidemic-no epidemic threshold, without resorting to special model forms. Nevertheless, this flexibility does not come without a cost in the development phase.

A brief overview of ANNs. ANNs are a family of information processing models whose original inspiration was a mathematical

Corresponding author: L. J. Francl; E-mail address: LFrancl@psu.edu

Publication no. P-2004-0719-030

(c) 2004 The American Phytopathological Society model of neuron activation given by the equation of McCulloch and Pitts (16):

$$
n_{j}(t)=I\left(\sum_{i \rightarrow j} w_{i j} n_{i}(t-1)>\theta_{i}\right)
$$

This equation represents the state of a neuron, $n_{j}$, at time, $t$, as the additive result of $n_{i}$ neural connections passing their activation states, which are attenuated by specific weights, $w_{i j}$, an inhibition factor $(I)$, and a threshold activation level $\left(\theta_{i}\right)$. In neural network terminology, transfer functions sometimes are called activation functions because if their value is above a given threshold, the receiving node is stimulated to "fire" (i.e., pass on binary numeric information). The next major advancement (24) was a feedback function that adjusted weights to minimal error values (Fig. 1); however, popularization of ANNs as a distinct class of models occurred in the 1980s (19) when the activation threshold, $\theta$, was replaced by a continuous function and a multilayer network took derivatives from a backpropagation of errors to approximate the target output by nonlinear functions (Fig. 2). This feed-forward backpropagation architecture has remained a mainstay among the models in the ANN family.

For further information on theory and methods, see texts authored by Bishop (1), Fausett (10), Ripley (18), and Smith (21) as well as searchable Internet resources and an electronic primer (20) updated monthly by the SAS Institute (Cary, NC).

Description of statistical ANNs. Several ANN forms have statistical properties in that they predict or classify empirical data with a known result $(2,23)$. The remainder of this article will focus on the feed-forward backpropagation neural network (BPNN) and the generalized regression neural network (GRNN), which are similar to statistical methods that estimate a function from empirical data (Table 1). Nonlinear regression models and feed-forward BPNNs both minimize error iteratively by the same method. The GRNN belongs to the probabilistic neural network class and is a direct correspondent of the nonparametric kernel regression technique $(17,22)$. Kernel methods predict a response by estimating the influence of known data cases within some proximity. For GRNN, the prediction is based on the computation of the most probable value of the dependent variable based on the consensus neighborhood of independent variables. 
ANN models in general consist of three features: (i) architecture or topology, (ii) learning rules or algorithms, and (iii) processing parameters. Each of these components is described briefly, followed by specifics for the two models of interest. Detailed explanations for these and other models are available $(1,10,18,20)$.

Network architecture. The basic architecture of an ANN model consists of nodes organized into an input layer, an output layer, and one or more intermediate layers between the input and output layers (Fig. 2). Each layer in the network contains one or more processing elements or nodes where numbers are temporarily stored. Typically, each input and output variable in the problem set are assigned to a single node and the intermediate layer will have a variable or assigned number of nodes. Input nodes typically are fully connected to the next layer by adaptive weights. Intermediate nodes summarize the various mathematical transfer functions, optionally apply a transformation, and pass the results to the output layer. Results are summarized in the output nodes, which correspond to one or more dependent variables.

The architecture of a BPNN can be exemplified as in Figure 3 from the Stagonospora leaf blotch prediction model (8). Disease incidence was predicted by five inputs and one intermediate layer of six nodes. Multiple intermediate layers are permitted within this class of models, but adding more nodes to a single layer can essentially duplicate any improvement due to this added complexity $(1,18)$. Feed-forward, often attached to descriptions of BPNN architecture, refers to the absence of internal feedback loops or within-layer connections. Two BPNN topological features not shown in Figure 3 are the backpropagated error and the bias node, which serves as the intercept term.

In GRNN, each set of input observations, $I_{1}$ to $I_{i}$, is associated one-to-one with an intermediate node, forming a pattern layer (Fig. 4). In the pattern layer, the values are processed according to the GRNN algorithm (described below). In a binary problem such

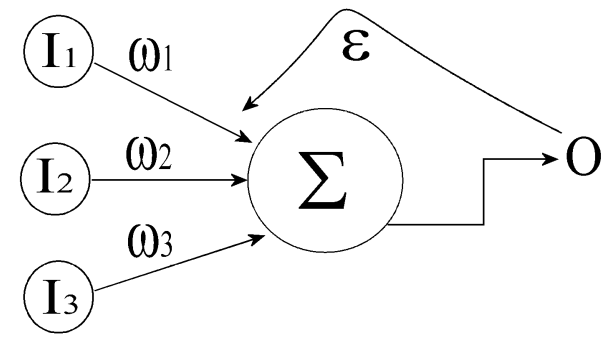

Fig. 1. The adaptive linear element or ADALINE of Widrow and Hoff (24) that approximates a desired output $(\mathrm{O})$ from input data (I) by optimally adjusting weights $(\omega)$ via an iterative error function $(\varepsilon)$.

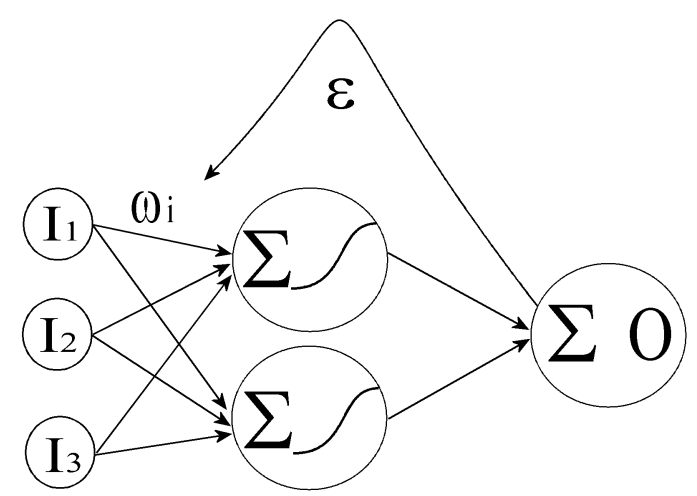

Fig. 2. The feed-forward, multilayer neural network with weighted $(\omega)$ numerical input information $(I)$ and a bias term (not shown) passed to an intermediate layer, summed and transformed by a sigmoidal function within that layer, and passed to the output node where information is again collected and summed. Predicted $(\mathrm{O})$ and observed values are compared during training and errors $(\varepsilon)$ are backpropagated to adjust individual weights. as epidemic-no epidemic, two nodes in a summary layer represent the possible outcomes and the output $(\mathrm{O})$ is the ratio of these two summary nodes.

Network algorithms. Algorithms direct how information is processed within the neural network architecture. The most efficient algorithms for most applications, including plant disease forecasting, appear to be those where the model output is compared with the correct result. This is called a supervised network in neural network terminology; in contrast, unsupervised networks involve some form of dimensionality reduction by clustering like inputs (20).

The feed-forward backpropagation class of algorithms iteratively approximates a target output. Values of input variables are weighted, passed forward to the next node where all links are summarized and a bias term added, and then transformed and transferred forward until yielding an estimated output value. The difference between estimated and true outputs is evaluated, and error is minimized by adjusting linkage weights after each learning cycle. Between $10^{2}$ and $10^{6}$ iterations are common during BPNN training.

The GRNN estimates the probability density function for each output pattern in the same manner as the Nadaraya-Watson kernel regression procedure $(17,18)$. The predicted value of a class density is the weighted average of the target values of training pat-

TABLE 1. Correspondence of artificial neural networks with statistical procedures $^{y}$

\begin{tabular}{ll}
\hline Network architecture & Statistical procedure \\
\hline Feed-forward without an intermediate layer & Generalized linear model ${ }^{\mathrm{z}}$ \\
Feed-forward with one intermediate layer & Projection pursuit regression \\
Probabilistic neural networks & Kernel discriminant analysis \\
Kohonen networks & $k$-means cluster analysis \\
Hebbian learning & Principal component analysis \\
\hline
\end{tabular}

y Degree of correlation ranges from similar to identical.

${ }^{\mathrm{z}}$ Intrinsically linear when the transfer function is sigmoid.

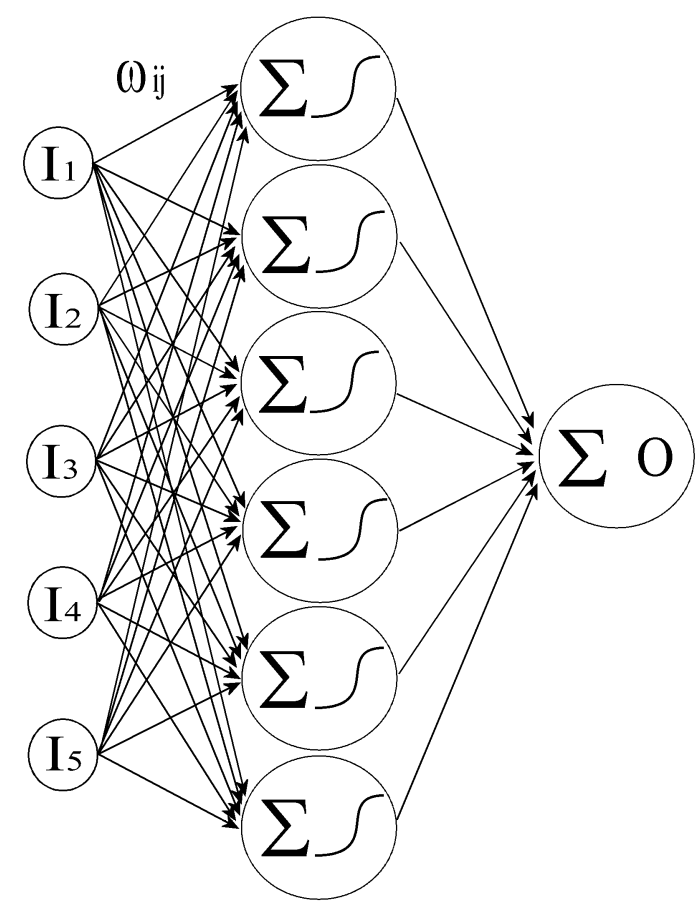

Fig. 3. The backpropagation neural network model architecture as implemented by De Wolf and Francl (8) to predict conduciveness for Stagonospora infection. Five standardized inputs $\left(I_{1}\right.$ to $\left.I_{5}\right)$ and a bias term $\left(I_{0}\right.$ not shown) were weighted individually (denoted by $\omega_{i j}$ ) and summarized in six intermediate nodes, and then a tanh function was taken and information passed to the output node, where values were again summarized to make the prediction $(\mathrm{O})$. 
terns close to the given output pattern $(4,22)$. Pattern similarity, i.e., degree of closeness, is defined by a smoothing factor, $\sigma$ (Fig. 4 ). The overall class density function receives a weight of 1.0 and serves as the denominator in the summation step. The individual pattern is weighted by $y_{i}$ and becomes the numerator. The output is scaled between 0.0 and 1.0 with class membership typically evaluated at a threshold of 0.5 . Since this is a probability density function, the modeler has the option of applying intermediate categories as a method of denoting confidence in the prediction.

Network parameterization. Whereas algorithms set the rules, processing parameters determine the details of how numerical information flows through the network. Parameters and rules of a network are inexorably intertwined; however, it may be useful to consider separately those elements that may be altered during the modeling exercise (e.g., by trial and error) without changing the fundamental type of network.

In a BPNN, transfer weights applied to the input variables may be initialized as either random or preset numbers. After summarization at the node, numerical information is transferred by a linear or sigmoidal function. The linear function often simply means the value is untransformed. Sigmoidal transformations can be a symmetrical logistic function ( 0.0 to 1.0 scale), hyperbolic (tanh with a -1.0 to 1.0 scale), or nonsymmetrical function (e.g., Gompertz).

While other methods are available, BPNN prediction error typically is evaluated by the method of least squares $(18,19)$. The backpropagation step adjusts weights to new values by taking a partial derivative of previous weights with respect to error. The new weights can be adjusted further to either speed or slow the process of iterative convergence.

Overfitting of training data is a critical modeling issue in BPNN development because of its impact on the model's ability to generalize to new data (Fig. 5A and B); accordingly, iteration is stopped after a set number of iterations, a convergence criterion that measures stepwise model error reduction, or some form of validation. A standard cross validation procedure is the "leave one out" computation, wherein every case is omitted iteratively and an estimate is derived from the remaining data. Another widely accepted method is to divide the data set into two, using the first for training and reserving the second for validation.

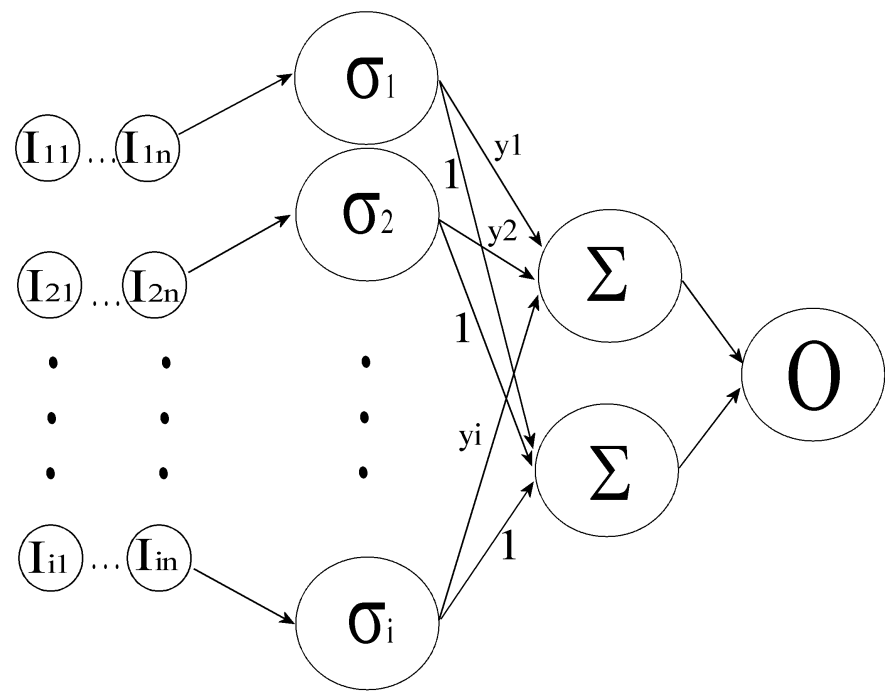

Fig. 4. The generalized regression neural network architecture showing the input variables $I_{11}$ to $I_{\text {in }}$ within empirical cases $I_{1}$ to $I_{i}$. The pattern layer has a single node associated with each case, a smoothing factor $(\sigma)$ applied as part of the density function, and the coefficient $y_{i}$ that produces the numerator for the summation layer. The denominator in the summation layer is the mean density function summed across all the empirical cases. The output divides the two values of the summation layer to yield the predicted dependent variable.
For GRNN modeling, the degree of smoothing greatly affects how accurately the network approximates the target output distribution, primarily through its effect on where to cut off the tails of the distribution, that is, the width of smoothing factor $\sigma$ (Fig. 5C and D). The GRNN algorithm can allow the modeler to set a $\sigma$, estimate a single $\sigma$ for the whole data set, or associate a separate $\sigma$ with each input variable $(3,5)$. Although the last approach is the most computationally intensive, the importance of each predictor variable can be taken into account. Conceptually, the radius of influence is optimized to avoid a compromised estimate (Fig. 5D).

Recommendations for application of ANNs in botanical epidemiology. Preparation. ANNs are commonly perceived as black boxes that lack customary scientific rigor. There is, in fact, a chaotic component to the nonlinear constructs that results from thousands of computational iterations initiated from a random value; moreover, it is virtually impossible to deduce from ANNs formal knowledge (e.g., rules of system behavior) or statistical properties (e.g., tests of hypotheses). However, several popular ANN architectures correspond directly to statistical models (Table 1) and therefore are subject to the same constraints regarding assumptions and remedial measures. The notion of a black box model may also lead to an expectation of exceedingly low error rates; rather, improvement in prediction accuracy of ANNs over appropriate statistical models seems to be incremental $(3,8)$.

An additional misconception stemming from the construction of a response surface without specification of the model form is that an ANN can find the optimal solution with little effort on the part of the modeler. In fact, execution of ANN development often requires a greater commitment of resources, not less. The BPNN in particular requires optimization of a set of parameters (number of intermediate nodes, type of transfer function, momentum term, etc.) by trial and error. A BPNN also may converge in a local minimum during iterative error gradient descent. Local error minima are unpredictable features of a nonlinear regression surface. Generally, a concerted effort is required to determine if an ANN has minimized global error; that is, whether it can optimally or at least near-optimally generalize from the training data set to predict a result from new examples.

Obviously, no model can extract patterns if the information does not exist a priori. Extraneous input variables will not help discern underlying patterns and, depending on the ANN model form, may seriously degrade performance (20). Moreover, a data set with too few training examples and an overly parameterized model (e.g., too many intermediate nodes) can lead to problems
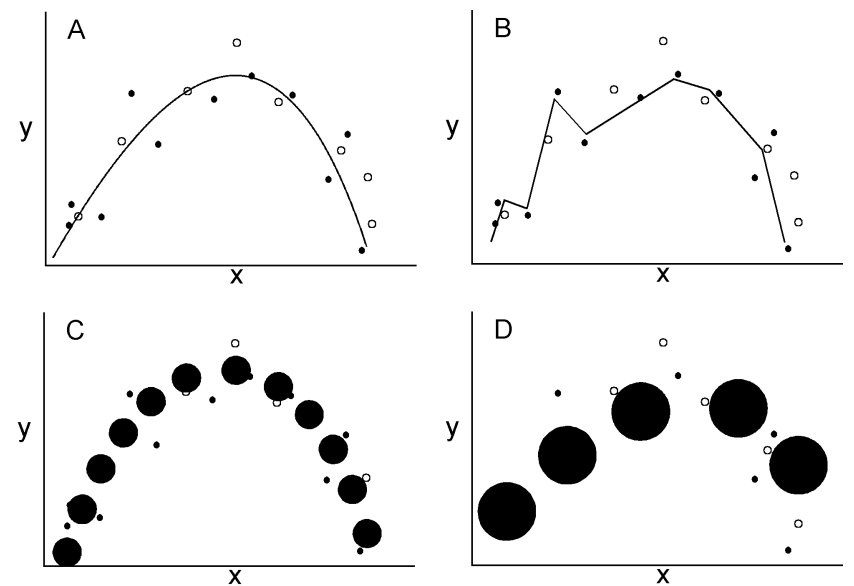

Fig. 5. Model fitting visualizations for arbitrary training (•) and validation (O) data points: A, appropriate stopping and parameterization for a backpropagation neural network; $\mathbf{B}$, overfitting by a backpropagation network produces a poorer fit to validation data; $\mathbf{C}$, a generalized regression neural network with an optimized smoothing parameter; and D, a generalized regression network with a smoothing parameter that is too large, producing a more diffuse area of influence and less responsive (i.e., flatter) function. 
with the ANN approach. Relatively simple relationships are better modeled with straightforward regression analysis and similar analytical methods. Similarly, ANNs should be reserved for situations where data contain at least moderately complex relationships.

Data set. The primary needs for disease forecasting with an ANN, as well as empirical modeling in general, are data that will adequately represent future conditions. Sample size requirements increase with model complexity and amount of random noise (21). Appropriate data subsets for model validation also increases data set size requirements. One also has to consider how well extreme values are represented and how the model will behave under conditions outside the range present in sample data. As a rule of thumb, data set size should be about triple the desired minimum size for correlation/regression analysis.

Preprocessing data. With any type of ANN, preconditioning data is a necessary preliminary step to satisfy distributional assumptions and assure convergence. Exploratory data analysis is an important early step toward understanding data distributions and the relationships among variables. Once variables are selected, they should be rescaled before presenting them to the network. This prevents overflow in transfer values (called saturation) and mitigates differences in scale among variables that might otherwise affect model performance. Normalization and the somewhat preferred standardization would collapse all variables to a comparable range of values (literature citation 20 provides calculations).

Preprocessing may also involve radical conditioning of input data, for example, formation of principal components and time series lag values. Both techniques have obvious potential application to plant disease forecasting. To avoid the risk of multicollinearity, for example, all hydric variables might be combined into one or a few principal components before presentation to a network.

Model specification. Some ANNs, such as GRNN, have few adjustable parameters, while others, such as BPNN, have so many conditional parameters that an analysis of all possible network settings is not pragmatic. The number of intermediate nodes and the transfer function have an especially large impact on BPNN performance. With regard to intermediate nodes, the principle of Occam's razor should be applied; that is, the simple model is to be preferred to the complex. Sarle (20) recommends starting with zero or one intermediate node and measuring model performance under standard conditions as more nodes are added. On standard test data sets, the hyperbolic transfer function (tanh) performed slightly better than the logistic, while the linear function ranked a distant third (20).

Although not a strict requirement for the development effort, a sensitivity analysis, which examines inputs in a piecewise fashion, can assess what information is most important to the prediction. A sensitivity analysis also can serve as a guide to removal of inconsequential input variables.

Model validation. Perhaps no step is quite so crucial to ANN modeling success as a complete and thorough validation. The ability of ANNs to find patterns leaves them subject to overfitting data sets, that is, finding patterns that may exist solely in the training data. The precise steps taken may vary depending on software package, data set size, and similar factors. Excellent reviews and recommendations can be found in Bishop (1), Ripley (18), and Sarle (20).

The removal of training examples for validation sometimes presents a difficulty because empirical models perform less well if there are insufficient training cases. Generally, a 2:1 to 3:1 ratio of training to validation cases is recommended (21). However, the precise number needed in each division is dependent on the problem's characteristics; i.e., random noise and functional complexity. The bootstrap method enhances small data sets by random assignment of cases to the validation subset in replicated samples.
For example, De Wolf and Francl (8) randomly assigned 152 cases to the training subset and 50 to the test subset in 10 replicated trials to assess mean model performance.

The hold out method of validation splits the data into training and validation sets. The ANN trains as usual but periodically checks the model against the validation set during training to prevent overfitting the model. However, the optimum model found by this method may have had a biased estimate of global error because true independence is lacking $(1,7,12)$. Bishop (1) and Smith (21) recommend a third data subset to validate the model in the usual sense if using the hold out method.

A case history of the North Dakota State University Small Grain Disease Forecasting System. Prediction of an impending plant disease epidemic can increase appropriate pesticide use, agricultural productivity, and returns to management. Key elements of a successful forecasting system include accurate prediction for multiple and economically important plant diseases, timely and understandable communication, and cost-effective remedies. Most existing forecasters are devoted to high-value crops because of the large reward for sound disease management in those cropping systems. Many of these systems consist of self-contained computer programs and environmental loggers positioned on-site. In contrast to high-value crops, few forecasters for field crops exist despite the large aggregate amounts of pesticides that may be applied. System development is hindered by the narrow return to added disease management expense and the relative economic inefficiency of an on-site system.

A spring wheat forecasting system was developed in three parallel research tracks, which are detailed in the following sections. The forecasting system was deployed in North Dakota and Minnesota in 1999. Tan spot, Stagonospora leaf blotch, Fusarium head blight were originally forecasted and wheat leaf rust was added the following year. The system comprised environmental and aerobiota input data, computer and rule-based models to predict disease, automated compilation of results, and information delivery via the Internet and toll-free number.

Wetness duration prediction. Obviously, moisture on host surfaces favors infection and reproduction by many plant pathogens. Disease forecast models thus need as input, duration of leaf wetness or surrogate relative humidity data, although surprisingly little attention has been paid to modeling wetness per se (14). To forecast infection period for wheat foliar diseases, moisture on plant surfaces should be predicted to within 1 to $2 \mathrm{~h}$ of the true duration $(13,14)$.

Input environmental data for the model came from an automated datalogger (Campbell Scientific, Logan, UT) as described elsewhere $(3,12)$. The first modeling effort (12) involved a comparison of a BPNN (Neuralworks Software, NeuralWare, Pittsburgh, PA) with logistic regression and multivariate linear discrimination (SAS Institute). The BPNN had the highest accuracy (93\% versus 87 to $90 \%$ ), but this estimate was derived from the hold out method, which may have been biased. When compared with several published dew prediction models, the BPNN was less accurate than a classification-regression tree approach (13) but more accurate than a physical model and relative humidity index. The BPNN model had an average error of $1.1 \mathrm{~h}$, which was within the specified accuracy goal.

The GRNN was evaluated in a second round of modeling wetness duration $(3,4)$. The GRNN has several advantages over the BPNN, including greater understanding of model results (i.e., repeatable derivation of coefficients), less reliance on parameter optimization since only $\sigma$ is adjustable, and less likelihood of being trapped in local minima during error gradient descent. Custom software was written to compare GRNN with four linear regression methods (5). The four linear regression methods provided approximately the same level of accuracy for test and validation data sets. As might be expected for a robust nonlinear method, the GRNN outperformed the linear methods by about 
$30 \%$ (3). The global error estimate was about $1.5 \%$ less than an earlier estimate (12), suggesting a better ANN implementation.

Overall, the GRNN predicted flag leaf moisture from dew and rain with greater than $90 \%$ accuracy. Inputs were available from the automated North Dakota Agricultural Weather Network (NDAWN); therefore, a regionally deployed forecaster could use this estimate in lieu of measured moisture. However, predictions were specific to only the flag leaf layer of a spring wheat canopy. Extension to other crops, geographic regions, or indeed lower leaf layers of wheat would require more empirical testing and modification.

Infection period prediction. The infection period models for tan spot and Stagonospora leaf blotch were modeled by BPNNs using an infection period bioassay $(7,8,11)$. A preliminary analysis $(7)$ focused on tan spot prediction by the hold out validation method; therefore, results will not be discussed further. A later analysis (8) included a prediction of Stagonospora leaf blotch, used a bootstrap validation method (18), and had a larger data set $(n=202)$ than the earlier effort. These analyses used Neuralworks for BPNN, software by Chtioui et al. (5) for GRNN, and SAS procedures for remaining statistical models. Four linear and nonlinear statistical models, the GRNN, and the BPNN were compared for accuracy (Table 2). For tan spot incidence, the BPNN slightly outperformed other models on average but differences were not statistically significant, whereas for Stagonospora leaf blotch, the BPNN was significantly more accurate. De Wolf and Francl (8) chose the single BPNN for each disease that performed most accurately on both training and validation data sets for further analysis. The selected BPNN for tan spot predicted $78 \%$ of the training and $82 \%$ of the validation cases and had seven intermediate nodes. The BPNN for Stagonospora leaf blotch predicted $85 \%$ of the training and $84 \%$ of the validation cases and had six intermediate nodes (Fig. 3).

The network architectures suggested that a similar level of input dimensionality reduction occurred for both models. A sensitivity analysis indicated that leaf wetness duration and cumulative growing degree-days were most important for tan spot prediction, whereas precipitation and relative humidity were most important for Stagonospora leaf blotch prediction. An error analysis for tan spot showed nearly all false positives (8/9) and all but one error associated with crop growth stages near flag leaf emergence (when inoculum may have limited infection). False positive errors for Stagonospora blotch infection were equal to false negatives and corresponded with precipitation events and wetness durations of $\geq 10 \mathrm{~h}$, which again suggests that inoculum was a limiting factor. False negative errors were associated with either the lack of precipitation or $\leq 10-\mathrm{h}$ wetness duration.

System integration and deployment. Adaptation of infection period models into a disease management system required further research to determine the growth stage and disease intensity level to start the models. Timing of fungicide applications was re-

TABLE 2. Global estimate of prediction accuracies for statistical and artificial neural network models of wheat tan spot and Stagonospora leaf blotch infection periods $(8)$

\begin{tabular}{lcc}
\hline & \multicolumn{2}{c}{ Accuracy $^{\mathrm{z}}$} \\
\cline { 2 - 3 } Model on validation data & Tan spot & Stagonospora $^{\mathrm{y}}$ \\
\cline { 2 - 3 } Linear discrimination & $72 \% \mathrm{a}$ & $75 \% \mathrm{~b}$ \\
Quadratic discrimination & $72 \% \mathrm{a}$ & $76 \% \mathrm{~b}$ \\
Logistic regression & $71 \% \mathrm{a}$ & $81 \% \mathrm{ab}$ \\
$k$-nearest neighbor & $74 \% \mathrm{a}$ & $76 \% \mathrm{~b}$ \\
Generalized regression neural network & $72 \% \mathrm{a}$ & $78 \% \mathrm{~b}$ \\
Backpropagation neural network & $76 \% \mathrm{a}$ & $86 \% \mathrm{a}$ \\
\hline
\end{tabular}

y Mean prediction accuracies of models are based on 10 randomized divisions of 202 cases into training and validation subsets. Percentages within a column followed by the same letter are not significantly different at $P<0.05$.

${ }^{\mathrm{z}}$ Infection is determined by healthy wheat plants exposed to outdoor wheat field environment for $24 \mathrm{~h}(11)$. searched in replicated trials wherein locally systemic fungicides seemed to be better candidates for integration into a forecasting system than protectant fungicides. Replicated trials with spring wheat during several years and in multiple North Dakota locations resulted in a foliar disease control advisory that involved scouting for a $50 \%$ disease incidence threshold on either the penultimate or antepenultimate leaf $(6,15)$.

The North Dakota State University forecasting system was deployed in North Dakota and Minnesota in 1999 for tan spot, Stagonospora leaf blotch, and Fusarium head blight of wheat. Tan spot and Stagonospora leaf blotch infection periods were predicted by BPNNs using environmental data from 17 automated weather stations of the NDAWN system. The GRNN predicted wetness duration as an additional input not available from the weather stations. The Fusarium head blight forecast was based on spore counts from a Burkard cyclonic sampler placed in a field of wheat stubble near the NDAWN station.

The system was expanded in each of the subsequent seasons until the system encompassed NDAWN in its entirety (63 locations in 2002). For the 2002 season, spore counts of Gibberella zeae were available from 21 locations. Importantly, forecasts from two Fusarium head blight models (9) were added in 2002, and information content on the website was enhanced with contour maps and other technologies. Taken collectively, continued experimentation, user feedback, and interface enhancement have been crucial to sustained operation of the forecasting system and its perceived value.

\section{EPILOGUE}

In multiple trials, ANN models have been equal or superior to comparable statistical models. The GRNN appears best suited for problems with many training examples, whereas BPNN seems more adept at modeling with fewer examples. However, a rigorous search for optimal BPNN network parameterization is time consuming and improvement in prediction accuracy over comparable statistical models may not always be enough to justify the effort. Many plant disease phenomena, in addition to disease forecasting, may be candidates for ANN models. For efficient resource allocation, ANNs should be applied to at least moderately complex epidemiological phenomena in an attempt to improve incrementally upon results achievable by mainstream statistical methods.

\section{LITERATURE CITED}

1. Bishop, C. M. 1995. Neural Networks for Pattern Recognition. University Press, Oxford, UK.

2. Cheng, B., Titterington, D. M. 1994. Neural networks: A review from a statistical perspective. Stat. Sci. 9:2-54.

3. Chtioui, Y., Francl, L. J., and Panigrahi, S. 1999. Moisture prediction from simple micrometeorological data. Phytopathology 89:668-672.

4. Chtioui, Y., Panigrahi, S., and Francl, L. 1998. A generalized regression neural network and its application for leaf wetness prediction to forecast plant disease. Chemom. Intel. Lab. Syst. 48:55-67.

5. Chtioui, Y., Panigrahi, S., and Francl, L. 1999. Neuro-Regress: Selected Parametric and Nonparametric Regression Tools for Chemometrics. Technical Description and Software Users Guide. North Dakota State University, Fargo.

6. De Wolf, E. D. 2000. Development and application of infection period models for wheat tan spot and Stagonospora blotch. Ph.D. thesis. North Dakota State University, Fargo.

7. De Wolf, E. D., and Francl, L. J. 1997. Neural networks that distinguish infection periods of tan spot of wheat in an outdoor environment. Phytopathology 87:83-87.

8. De Wolf, E. D., and Francl, L. J. 2000. Neural network classification of tan spot and Stagonospora blotch infection periods in a wheat field environment. Phytopathology 90:108-113.

9. De Wolf, E. D., Madden, L. V., and Lipps, P. E. 2003. Risk assessment models for wheat Fusarium head blight epidemics based on within-season weather data. Phytopathology 93:428-435.

10. Fausett, L. 1994. Fundamentals of Neural Networks: Architectures, Al- 
gorithms, and Applications. Prentice Hall, Englewood Cliffs, NJ.

11. Francl, L. J. 1995. Challenge of bioassay plants in a monitored outdoor environment. Can. J. Plant Pathol. 17:138-143.

12. Francl, L. J., and Panigrahi, S. 1997. Artificial neural network models of wheat leaf wetness. Agric. For. Meteorol. 88:57-65.

13. Gleason, M. L., Taylor, S. E., Loughlin, T. M., and Koehler, K. J. 1994. Development and validation of an empirical model to estimate the duration of dew periods. Plant Dis. 78:1011-1016.

14. Huber, L., and Gillespie, T. J. 1992. Modeling leaf wetness in relation to plant disease epidemiology. Annu. Rev. Phytopathol. 30:553-577.

15. Larson, C. 2001. Evaluation of the NDSU wheat disease forecasting system. MS thesis. North Dakota State University, Fargo.

16. McCulloch, W. S., and Pitts, W. 1943. A logical calculus of ideas immanent in neural activity. Bull. Math. Phys. 5:115-133.

17. Nadaraya, E. A. 1964. On estimating regression. Theory Prob. Appl. 9:141-142.
18. Ripley, B. D. 1996. Pattern Recognition and Neural Networks. University Press, Cambridge, UK.

19. Rumelhart, D. E., Hinton, G. E., and Williams, R. J. 1986. Learning representation by back-propagating errors. Nature 323:533-536.

20. Sarle, W. S. 2002. Neural network FAQ: Introduction. Documented online as FAQ 1473 by the SAS Institute, Cary, NC.

21. Smith, M. 1996. Neural Networks for Statistical Modeling. International Thomson Computer Press, Boston, MA

22. Specht, D. F. 1991. A general regression neural network. IEEE Trans. Neural Networks 2:568-576.

23. White, H. 1989. Learning in artificial neural networks: A statistical perspective. Neural Comput. 1:425-464.

24. Widrow, B., and Hoff, M. E., Jr. 1960. Adaptive switching circuits. IRE WESCON Convention Record, part 4, Pages 96-104 [Reprinted in 1988: Neurocomputing: Foundations of Research. J. A. Anderson and E. Rosenfeld, eds. MIT Press, Cambridge, MA]. 